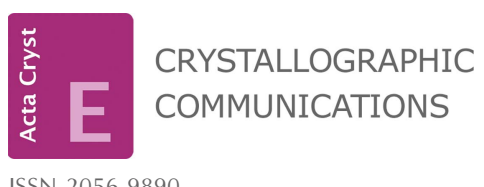

ISSN 2056-9890

\section{Solvent inclusion in the crystal structure of bis[(adamantan-1-yl)methanaminium chloride] 1,4-dioxane hemisolvate monohydrate explained using the computed crystal energy landscape}

Sharmarke Mohamed*

Khalifa University, PO BOX 127788, Abu Dhabi, United Arab Emirates. *Correspondence e-mail: sharmarke.mohamed@kustar.ac.ae

Repeated attempts to crystallize 1-adamantanemethylamine hydrochloride as an anhydrate failed but the salt was successfully crystallized as a solvate $\left(2 \mathrm{C}_{11} \mathrm{H}_{20} \mathrm{~N}^{+} \cdot 2 \mathrm{Cl}^{-} \cdot 0.5 \mathrm{C}_{4} \mathrm{H}_{8} \mathrm{O}_{2} \cdot \mathrm{H}_{2} \mathrm{O}\right)$, with water and 1,4 -dioxane playing a structural role in the crystal and engaging in hydrogen-bonding interactions with the cation and anion. Computational crystal-structure prediction was used to rationalize the solvent-inclusion behaviour of this salt by computing the solvent-accessible voids in the predicted low-energy structures for the anhydrate: the global lattice-energy minimum structure, which has the same packing of the ions as the solvate, has solvent-accessible voids that account for $3.71 \%$ of the total unit-cell volume and is $6 \mathrm{~kJ} \mathrm{~mol}^{-1}$ more stable than the next most stable predicted structure.

\section{Chemical context}

The rational synthesis of multi-component crystal forms using hydrogen-bond synthons (Desiraju, 1995) between donor and acceptor groups (Duggirala et al., 2015) to direct the threedimensional assembly of two or more molecules in the solid state is an active area of crystal-engineering research. In recent years, there has been significant progress (Reilly et al., 2016) in computational methods for predicting the most stable crystal structures of multi-component salt and co-crystal solid forms using only the molecular structures as input. By comparison, the challenge of predicting when some molecules will crystallize as solvates has received little attention (Braun et al., 2013) from the crystal-engineering community and, despite evidence (Aakeröy et al., 2007) from the Cambridge Structural Database (Groom et al., 2016) that salt solid forms are more prone to crystallizing in structures with variable compositions and stoichiometries, the underlying factors behind the crystallization of salt solvates and the rational synthesis of such solid forms remains an under-explored area of crystal-engineering research. Previous work on the solventinclusion behaviour of substituted adamantane hydrochloride salts (Mohamed et al., 2016) has shown that mapping the percentage solvent-accessible volumes of predicted lowenergy structures can provide a qualitative assessment of the likelihood of crystallizing non-stoichiometric channel hydrates of hydrochloride salts. In this work, the computational model is extended to rationalize the solvent-inclusion behaviour of 1-adamantanemethylamine hydrochloride on the basis of the packing efficiency of the ions and calculated solvent-accessible voids for the anhydrate. 

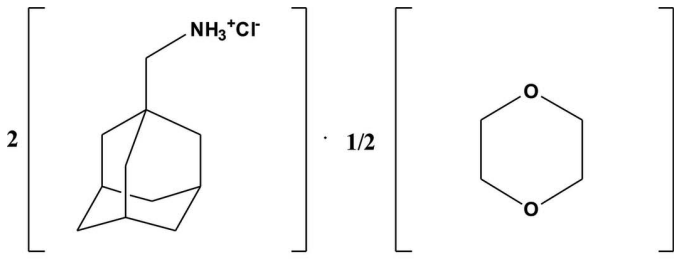

(I)
Table 1

Hydrogen-bond geometry $\left(\AA,^{\circ}\right)$.

\begin{tabular}{lllll}
\hline$D-\mathrm{H} \cdots A$ & $D-\mathrm{H}$ & $\mathrm{H} \cdots A$ & $D \cdots A$ & $D-\mathrm{H} \cdots A$ \\
\hline $\mathrm{O} 1-\mathrm{H} 1 D \cdots \mathrm{Cl} 2^{\mathrm{i}}$ & $0.82(3)$ & $2.48(3)$ & $3.295(2)$ & $175(3)$ \\
$\mathrm{O} 1-\mathrm{H} 1 E \cdots \mathrm{Cl} 1$ & $0.91(4)$ & $2.36(4)$ & $3.265(2)$ & $180(3)$ \\
$\mathrm{N} 1-\mathrm{H} 1 A \cdots \mathrm{Cl} 2^{\mathrm{ii}}$ & $0.85(3)$ & $2.33(3)$ & $3.161(2)$ & $163(2)$ \\
$\mathrm{N} 1-\mathrm{H} 1 B \cdots \mathrm{O} 2$ & $0.89(3)$ & $2.10(3)$ & $2.867(3)$ & $144(2)$ \\
$\mathrm{N} 1-\mathrm{H} 1 C \cdots \mathrm{Cl} 1$ & $0.98(3)$ & $2.19(3)$ & $3.152(2)$ & $166(2)$ \\
$\mathrm{N} 2-\mathrm{H} 2 C \cdots \mathrm{Cl} 2^{\mathrm{i}}$ & $0.86(2)$ & $2.31(3)$ & $3.166(2)$ & $172(2)$ \\
$\mathrm{N} 2-\mathrm{H} 2 D \cdots \mathrm{Cl} 1$ & $0.86(3)$ & $2.48(3)$ & $3.171(2)$ & $138(2)$ \\
$\mathrm{N} 2-\mathrm{H} 2 E \cdots \mathrm{Cl} 2$ & $0.90(3)$ & $2.30(3)$ & $3.181(2)$ & $166(2)$ \\
\hline
\end{tabular}

Symmetry codes: (i) $-x+2,-y,-z+1$; (ii) $x-1, y, z$.

\section{Structural commentary}

The asymmetric unit (Fig. 1) of the title structure (I) consists of two formula units of 1-adamantanemethylamine hydrochloride, half a molecule of 1,4-dioxane and one water molecule. Both cations adopt a rigid conformation due to the adamantane ring and an overlay of the $a b$ initio gas-phaseoptimized conformation of the cation at the MP2/6-31G(d,p) level of theory with the experimental conformation of each symmetry-unique cation revealed a root-mean-squared deviation of less than $0.03 \AA$ for the non-hydrogen atoms. The $\mathrm{C} 1-\mathrm{C} 11-\mathrm{N} 1$ and $\mathrm{C} 12-\mathrm{C} 22-\mathrm{N} 2$ bond angles for the cations are $113.76(17)$ and $113.72(16)^{\circ}$, which is consistent with the observation of identical molecular conformations. The 1,4-dioxane molecule lies on an inversion centre with $\mathrm{C} 23$ $\mathrm{O} 2$ and $\mathrm{C} 24-\mathrm{O} 2$ bond distances of 1.429 (3) and 1.425 (3) $\AA$, respectively.

\section{Supramolecular features}

All $\mathrm{N}^{+}-\mathrm{H}$ bond lengths (Table 1 ) are between 0.85 and $0.98 \AA$ and $\mathrm{N}^{+} \ldots \mathrm{Cl}^{-}$donor-acceptor distances are within the range 3.152-3.181 $\AA$, which is consistent with the hydrogen-bond geometries in related 1-aminoadamantane hydrochloride salts derived from primary amines such as adamantanamine hydrochloride (Bélanger-Gariépy et al., 1987) and (3,5-dimethyl-1-adamantyl)ammonium chloride hydrate (Lou et al.,

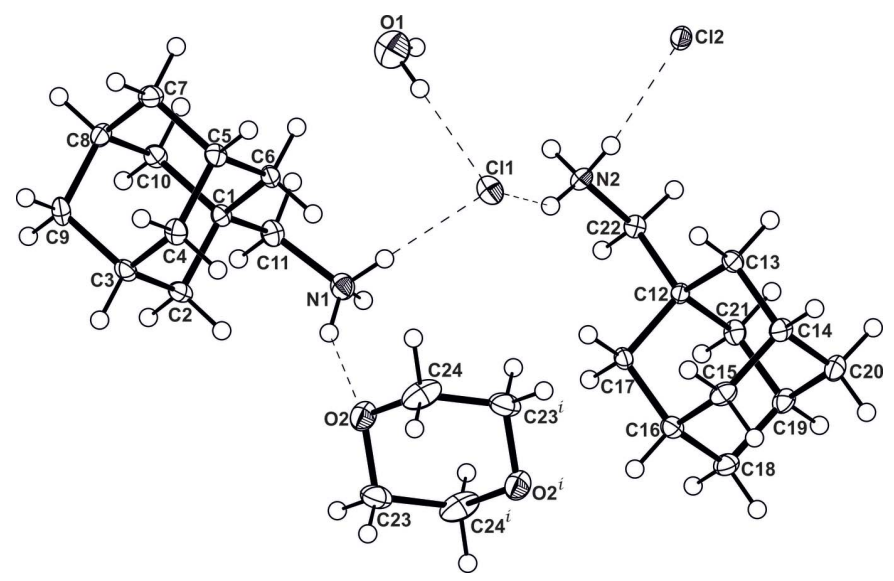

Figure 1

The molecular structure of (I) with displacement ellipsoids drawn at the $50 \%$ probability level and hydrogen atoms are shown as spheres of arbitrary radius. Symmetry code: (i) $1-x, 1-y, 1-z$.
2009). All $\mathrm{N}^{+}-\mathrm{H}$ hydrogen-bond donors on the cations engage in conventional hydrogen-bonding interactions with a chloride anion except for the $\mathrm{N}^{+}-\mathrm{H} 1 B \cdots \mathrm{O} 2$ hydrogen bond which involves the $\mathrm{O}$ atom of 1,4-dioxane as a hydrogen-bond acceptor. All hydrogen-bonding interactions involving the donor-acceptor pairs $\mathrm{N}^{+} \ldots \mathrm{Cl}^{-}$or $\mathrm{N}^{+} \ldots \mathrm{O}$ are characterized by discrete interactions of graph set $D_{1}^{1}(2)$. The crystal packing (Fig. 2) consists of a pleated ribbon stacking of the symmetryinequivalent cations $\left(A^{+}\right.$and $\left.B^{+}\right)$of 1-adamantanemethylamine along the $a$ axis with a chloride ion hydrogen bonded to both symmetry-inequivalent cations in an infinite $A^{+} \cdots \mathrm{Cl}^{-} \cdots B^{+}$pattern. This pleated ribbon stacking of the ions is similar to that observed in the crystal structure of 1-aminoadamantane hydrochloride (Bélanger-Gariépy et al., 1987). In the title structure, each water molecule engages in discrete $\mathrm{O}-\mathrm{H} \cdots \mathrm{Cl}^{-}$hydrogen bonding interactions and each 1,4-dioxane molecule acts as a hydrogen-bond acceptor to the $\mathrm{N}^{+}-\mathrm{H}$ donor of the cation. Both solvent molecules occupy the voids between successive pleated ribbons formed from the stacking of hydrogen-bonded $\mathrm{N}^{+}-\mathrm{H} \cdots \mathrm{Cl}^{-}$charged units.

\section{Computed crystal energy landscape}

The computed crystal energy landscape (Fig. 3) of 1-adamantanemethylamine hydrochloride was used to assess the possibility of solvent inclusion for this salt by estimating

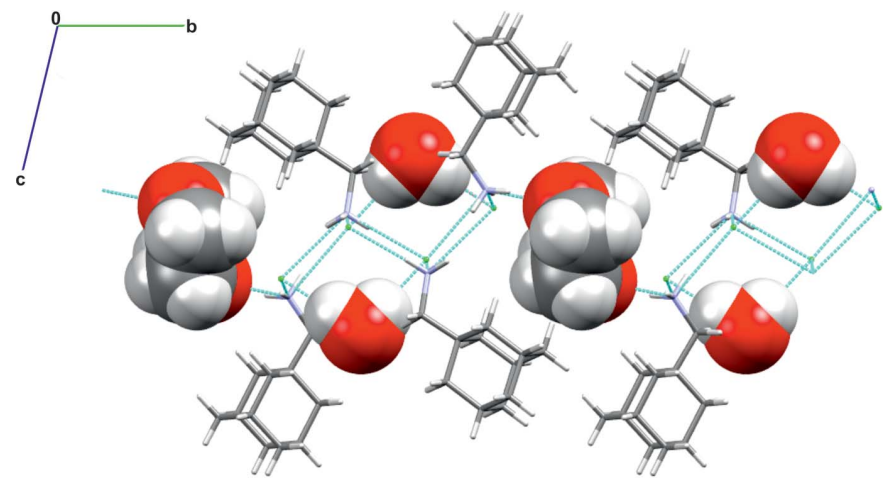

Figure 2

Crystal packing diagram for (I). The 1,4-dioxane and water molecules are shown using a space-filling model. Intermolecular hydrogen-bonding interactions are illustrated using blue dashed lines. 


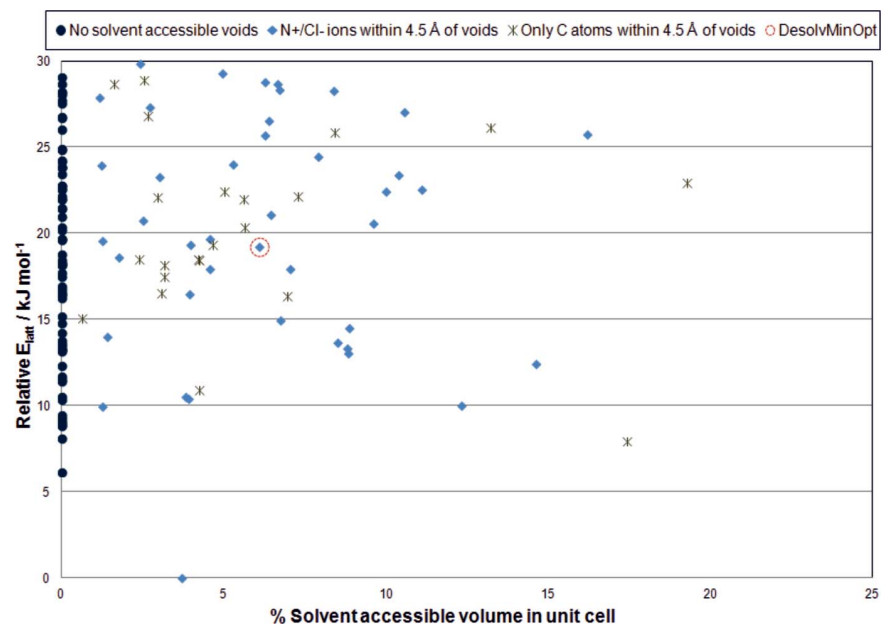

Figure 3

Predicted crystal energy landscape of (adamantan-1-yl)methanaminium chloride. The lattice energy is plotted relative to the predicted global minimum structure for the salt. The data point labelled DesolvMinOpt corresponds to the theoretical lattice energy minimum structure that would result from desolvation of the experimental (adamantan-1yl)methanaminium chloride 1,4-dioxane hydrate structure.

the percentage solvent-accessible volume in the predicted most stable packings. The most stable structure on the crystal energy landscape of the anhydrate displays a total potential solvent-accessible volume of $45.6 \AA^{3}$, which corresponds to $3.71 \%$ of the unit-cell volume. Assuming that each water molecule occupies an approximate total volume of $40 \AA^{3}$, this would suggest that the global minimum structure could be crystallized by dehydration of a monohydrate of the salt. The global lattice energy minimum structure is approximately $6 \mathrm{~kJ} \mathrm{~mol}^{-1}$ more stable than the nearest competing secondranked structure. The observation of a clearly preferred global lattice energy minimum structure with solvent-accessible voids is not conclusive in suggesting that this hydrochloride salt cannot be crystallized as an anhydrate, but it does suggest that this system will have difficulties crystallizing as an anhydrate since there is an energetic preference for a packing of the ions that is susceptible to solvent inclusion. Although the secondranked most stable predicted structure does not have any solvent-accessible voids, this structure is energetically competitive with the third-ranked structure which displays an unusually large percentage solvent-accessible volume of $17.42 \%$ of the unit cell. The majority of the predicted structures within $10 \mathrm{~kJ} \mathrm{~mol}^{-1}$ of the global minimum structure that have solvent-accessible voids have crystal voids that are located within $4.5 \AA$ of the charged $\mathrm{N}^{+} / \mathrm{Cl}^{-}$ions, which is consistent with the observation that both the water and 1,4dioxane solvent molecules in the experimental structure engage in hydrogen-bonding interactions with the $\mathrm{N}^{+}-\mathrm{H}$ donor and $\mathrm{Cl}^{-}$acceptor groups of the salt.

Although there is a clear thermodynamically preferred global minimum structure with solvent-accessible voids, the calculations also reveal that there are a number of energetically competitive packings of the ions within $10 \mathrm{~kJ} \mathrm{~mol}^{-1}$ of the global minimum structure that do not have solvent- accessible voids. However, $88 \%$ of these structures have one or two unused $\mathrm{N}^{+}-\mathrm{H}$ donors as judged by $\mathrm{N}^{+} \ldots \mathrm{Cl}^{-}$distances that are longer than the sum of the van der Waals radii of the $\mathrm{N}$ and $\mathrm{Cl}$ atoms, suggesting challenges in close packing of the ions which is consistent with the observation of solvent inclusion in this salt with solvent molecules engaged in hydrogen-bonding interactions. The structurally related 1-aminoadamantane molecule, which differs from 1-adamantanemethlyamine in that it lacks a methylene group bridging the adamantane ring and $\mathrm{NH}_{2}$ functional group displays a crystal energy landscape (Mohamed et al., 2016) with a single preferred global minimum structure corresponding to the experimentally observed anhydrate structure of the salt. This illustrates the sensitivity of crystal packing to minor modifications in molecular structure and the value of mapping the percentage solvent-accessible voids in predicted low-energy structures of hydrochlorides as a means for assessing the possibility of solvent inclusion.

\section{Database survey}

A search of the Cambridge Structural Database (CSD Version 5.37 plus three updates, no filters; Groom et al. 2016) has shown that there are no previously reported crystal structures of 1-adamantanemethylamine or multi-component salt or cocrystal structures of this primary amine. However, a dimethylformamide solvate of a platinum coordination complex involving two crystallographically inequivalent 1-adamantanemethylamine molecules coordinated onto platinum(II) metal has been reported (DUHKAT: Rochon \& Tessier, 2009) from crystallization experiments involving cisplatin [cis$\left.\mathrm{Pt}\left(\mathrm{NH}_{3}\right)_{2} \mathrm{Cl}_{2}\right]$ and 1-adamantanemethylamine in dimethylformamide. There are a number of reported crystal structures of substituted aminoadamantane hydrochloride salts such as 1-aminoadamantanamine hydrochloride (FINVAZ: BélangerGariépy et al., 1987), (3,5-dimethyl-1-adamantyl)ammonium chloride hydrate (DUCYAC: Lou et al., 2009) and (RS)-1-(1adamantyl)ethanamine hydrochloride (TOKWUN: Mishnev \& Stepanovs, 2014).

\section{Synthesis and crystallization}

A 1:5 ratio of HCl:acetone mixture was prepared and $0.3 \mathrm{ml}$ of 1-adamantanemethylamine was added to a vial containing $2 \mathrm{ml}$ of the HCl:acetone mixture. The contents of the vial were further diluted by adding $3 \mathrm{ml}$ of a 1:1 mixture of 1,4-dioxane: ethanol. The contents of the vial were shaken vigorously for two minutes and filtered under gravity. The solvent was allowed to evaporate under laboratory temperature and pressure conditions and after $24 \mathrm{~h}$ crystals of the title solvate with needle morphology were isolated.

\section{Refinement}

Crystal data, data collection and structure refinement details are summarized in Table 2. Hydrogen atoms attached to $\mathrm{N}$ and $\mathrm{O}$ atoms were located from difference Fourier maps and freely 
Table 2

Experimental details.

\begin{tabular}{|c|c|}
\hline \multicolumn{2}{|l|}{ Crystal data } \\
\hline Chemical formula & $2 \mathrm{C}_{11} \mathrm{H}_{20} \mathrm{~N}^{+} \cdot 2 \mathrm{Cl}^{-} \cdot 0.5 \mathrm{C}_{4} \mathrm{H}_{8} \mathrm{O}_{2} \cdot \mathrm{H}_{2} \mathrm{O}$ \\
\hline$M_{\mathrm{r}}$ & 465.53 \\
\hline Crystal system, space group & Triclinic, $P \overline{1}$ \\
\hline Temperature $(\mathrm{K})$ & 100 \\
\hline$a, b, c(\AA)$ & $6.4941(11), 13.491(2), 15.086(3)$ \\
\hline$\alpha, \beta, \gamma\left({ }^{\circ}\right)$ & $102.911(3), 91.824(3), 101.500(3)$ \\
\hline$V\left(\AA^{3}\right)$ & $1258.5(4)$ \\
\hline$Z$ & 2 \\
\hline Radiation type & Мо $K \alpha$ \\
\hline$\mu\left(\mathrm{mm}^{-1}\right)$ & 0.28 \\
\hline Crystal size $(\mathrm{mm})$ & $0.2 \times 0.05 \times 0.05$ \\
\hline \multicolumn{2}{|l|}{ Data collection } \\
\hline Diffractometer & Bruker APEXII CCD \\
\hline Absorption correction & $\begin{array}{l}\text { Multi-scan (SADABS; Bruker, } \\
\text { 2015) }\end{array}$ \\
\hline$T_{\min }, T_{\max }$ & $0.655,0.746$ \\
\hline $\begin{array}{l}\text { No. of measured, independent and } \\
\text { observed }[I>2 \sigma(I)] \text { reflections }\end{array}$ & $35014,6312,4285$ \\
\hline$R_{\text {int }}$ & 0.096 \\
\hline$(\sin \theta / \lambda)_{\max }\left(\AA^{-1}\right)$ & 0.670 \\
\hline \multicolumn{2}{|l|}{ Refinement } \\
\hline$R\left[F^{2}>2 \sigma\left(F^{2}\right)\right], w R\left(F^{2}\right), S$ & $0.056,0.105,1.06$ \\
\hline No. of reflections & 6312 \\
\hline No. of parameters & 303 \\
\hline $\mathrm{H}$-atom treatment & $\begin{array}{l}\mathrm{H} \text { atoms treated by a mixture of } \\
\text { independent and constrained }\end{array}$ \\
\hline$\Delta \rho_{\max }, \Delta \rho_{\min }\left(\mathrm{e} \AA^{-3}\right)$ & $0.49,-0.39$ \\
\hline
\end{tabular}

Computer programs: APEX2 and SAINT (Bruker 2015), XT (Sheldrick, 2015), SHELXL (Sheldrick, 2008) and OLEX2 (Dolomanov et al., 2009).

refined. All other hydrogen atoms were positioned geometrically $(\mathrm{C}-\mathrm{H}=0.99-1.00 \AA)$ and refined using a riding model with $U_{\text {iso }}(\mathrm{H})=1.2 U_{\text {eq }}($ carrier $)$.

\section{Computational modelling methodology}

The crystal energy landscape of 1-adamantanemethylamine hydrochloride was calculated using a search criterion that restricted crystal packings to those with one $\left(Z^{\prime}=1\right)$ or two $\left(Z^{\prime}\right.$ $=2$ ) formula units of the ions in the asymmetric unit using the Materials Studio 8.0 (Accelrys, 2014) code. Hypothetical crystal structures were generated in five of the most common space groups $\left(P \overline{1}, P 2_{1}, P 2_{1} / c, P 2_{1} 2_{1} 2_{1}, C 2 / c\right)$ for organic crystal structures using the MP2/6-31G(d,p) optimized geometry for the protonated 1-adamantanemethylamine cation. The atomic charges on the cation were derived by fitting to the molecular electrostatic potential of the optimized conformation using the ChelpG (Breneman \& Wiberg, 1990) scheme. The molecular geometry and fitted charges for the cation were calculated using GAUSSIAN09 (Frisch et al., 2009). The final lattice energies for the predicted structures were estimated using a distributed multipole model for the charges using DMACRYS (Price et al., 2010). Dispersion-repulsion contributions towards the lattice energy were estimated using the revised Williams99 force field (Williams, 2001) supplemented with the potential parameter set for the $\mathrm{Cl}^{-}$ion (Hejczyk, 2010). For all predicted structures in the crystal energy landscape, the solvent-accessible volume per unit cell was estimated using
PLATON (Spek, 2009) assuming a probe radius of $1.2 \AA$. Detailed settings for the Materials Studio 8.0 search for putative crystal structures and the DMACRYS lattice energy optimizations are the same as those reported in recent work (Mohamed et al., 2016) investigating the utility of computed crystal energy landscapes for inferring the risk of crystal hydration in substituted adamantane hydrochloride salts.

\section{Acknowledgements}

Professor Panče Naumov and Dr Durga Prasad Karothu of New York University Abu Dhabi are acknowledged for their support during the course of this research. Dr Liang Li is acknowledged for his support on data collection and structure solution. This research was carried out using Core Technology Platform resources at New York University Abu Dhabi.

\section{References}

Aakeröy, C. B., Fasulo, M. E. \& Desper, J. (2007). Mol. Pharm. 4, $317-$ 322.

Accelrys (2014). Materials Studio, Version 8.0. Accelrys Inc., San Diego, California, USA.

Bélanger-Gariépy, F., Brisse, F., Harvey, P. D., Butler, I. S. \& Gilson, D. F. R. (1987). Acta Cryst. C43, 756-759.

Braun, D. E., Bhardwaj, R. M., Arlin, J.-B., Florence, A. J., Kahlenberg, V., Griesser, U. J., Tocher, D. A. \& Price, S. L. (2013). Cryst. Growth Des. 13, 4071-4083.

Breneman, C. M. \& Wiberg, K. B. (1990). J. Comput. Chem. 11, 361373.

Bruker (2015). APEX2, SAINT and SADABS. Bruker AXS Inc., Madison, Wisconsin, USA.

Desiraju, G. R. (1995). Angew. Chem. Int. Ed. Engl. 34, 2311-2327.

Dolomanov, O. V., Bourhis, L. J., Gildea, R. J., Howard, J. A. K. \& Puschmann, H. (2009). J. Appl. Cryst. 42, 339-341.

Duggirala, N. K., Wood, G. P., Fischer, A., Wojtas, Ł., Perry, M. L. \& Zaworotko, M. J. (2015). Cryst. Growth Des. 15, 4341-4354.

Frisch, M. J., Trucks, G. W., Schlegel, H. B., Scuseria, G. E., Robb, M. A., Cheeseman, J. R., Scalmani, G., Barone, V., Mennucci, B., Petersson, G. A., Nakatsuji, H., Caricato, M., Li, X., Hratchian, H. P., Izmaylov, A. F., Bloino, J., Zheng, G., Sonnenberg, J. L., Hada, M., Ehara, M., Toyota, K., Fukuda, R., Hasegawa, J., Ishida, M., Nakajima, T., Honda, Y., Kitao, O., Nakai, H., Vreven, T., Montgomery, J. A., Jr., Peralta, J. E., Ogliaro, F., Bearpark, M., Heyd, J. J., Brothers, E., Kudin, K. N., Staroverov, V. N., Kobayashi, R., Normand, J., Raghavachari, K., Rendell, A., Burant, J. C., Iyengar, S. S., Tomasi, J., Cossi, M., Rega, N., Millam, J. M., Klene, M., Knox, J. E., Cross, J. B., Bakken, V., Adamo, C., Jaramillo, J., Gomperts, R., Stratmann, R. E., Yazyev, O., Austin, A. J., Cammi, R., Pomelli, C., Ochterski, J. W., Martin, R. L., Morokuma, K., Zakrzewski, V. G., Voth, G. A., Salvador, P., Dannenberg, J. J., Dapprich, S., Daniels, A. D., Farkas, Ø., Foresman, J. B., Ortiz, J. V., Cioslowski, J. \& Fox, D. J. (2009). GAUSSIAN09. Gaussian INC., Wallingford, CT, USA.

Groom, C. R., Bruno, I. J., Lightfoot, M. P. \& Ward, S. C. (2016). Acta Cryst. B72, 171-179.

Hejczyk, K. E. (2010). PhD thesis. University of Cambridge, UK.

Lou, W.-J., Hu, X.-R. \& Gu, J.-M. (2009). Acta Cryst. E65, o2191.

Mishnev, A. \& Stepanovs, D. (2014). Z. Naturforsch. Teil B, 69, 823828.

Mohamed, S., Karothu, D. P. \& Naumov, P. (2016). Acta Cryst. B72, 551-561.

Price, S. L., Leslie, M., Welch, G. W. A., Habgood, M., Price, L. S., Karamertzanis, P. G. \& Day, G. M. (2010). Phys. Chem. Chem. Phys. 12, 8478-8490. 
Reilly, A. M., Cooper, R. I., Adjiman, C. S., Bhattacharya, S., Boese, A. D., Brandenburg, J. G., Bygrave, P. J., Bylsma, R., Campbell, J. E., Car, R., Case, D. H., Chadha, R., Cole, J. C., Cosburn, K., Cuppen, H. M., Curtis, F., Day, G. M., DiStasio, R. A. Jr, Dzyabchenko, A., van Eijck, B. P., Elking, D. M., van den Ende, J. A., Facelli, J. C., Ferraro, M. B., Fusti-Molnar, L., Gatsiou, C.-A., Gee, T. S., de Gelder, R., Ghiringhelli, L. M., Goto, H., Grimme, S., Guo, R., Hofmann, D. W. M., Hoja, J., Hylton, R. K., Iuzzolino, L., Jankiewicz, W., de Jong, D. T., Kendrick, J., de Klerk, N. J. J., Ko, H.-Y., Kuleshova, L. N., Li, X., Lohani, S., Leusen, F. J. J., Lund, A. M., Lv, J., Ma, Y., Marom, N., Masunov, A. E., McCabe, P., McMahon, D. P., Meekes, H., Metz, M. P., Misquitta, A. J., Mohamed, S., Monserrat, B., Needs, R. J., Neumann, M. A., Nyman,
J., Obata, S., Oberhofer, H., Oganov, A. R., Orendt, A. M., Pagola, G. I., Pantelides, C. C., Pickard, C. J., Podeszwa, R., Price, L. S., Price, S. L., Pulido, A., Read, M. G., Reuter, K., Schneider, E., Schober, C., Shields, G. P., Singh, P., Sugden, I. J., Szalewicz, K., Taylor, C. R., Tkatchenko, A., Tuckerman, M. E., Vacarro, F., Vasileiadis, M., Vazquez-Mayagoitia, A., Vogt, L., Wang, Y., Watson, R. E., de Wijs, G. A., Yang, J., Zhu, Q. \& Groom, C. R. (2016). Acta Cryst. B72, 439-459.

Rochon, F. D. \& Tessier, C. (2009). Acta Cryst. E65, m1297-m1298.

Sheldrick, G. M. (2008). Acta Cryst. A64, 112-122.

Sheldrick, G. M. (2015). Acta Cryst. C71, 3-8.

Spek, A. L. (2009). Acta Cryst. D65, 148-155.

Williams, D. E. (2001). J. Comput. Chem. 22, 1154-1166. 


\section{supporting information}

Acta Cryst. (2016). E72, 1348-1352 [https://doi.org/10.1107/S2056989016013256]

Solvent inclusion in the crystal structure of bis[(adamantan-1-

yl)methanaminium chloride] 1,4-dioxane hemisolvate monohydrate explained using the computed crystal energy landscape

\section{Sharmarke Mohamed}

Computing details

Data collection: APEX2 (Bruker 2015); cell refinement: SAINT (Bruker 2015); data reduction: SAINT (Bruker 2015); program(s) used to solve structure: XT (Sheldrick, 2015); program(s) used to refine structure: SHELXL (Sheldrick, 2008); molecular graphics: OLEX2 (Dolomanov et al., 2009); software used to prepare material for publication: $O L E X 2$ (Dolomanov et al., 2009).

Bis[(adamantan-1-yl)methanaminium chloride] 1,4-dioxane hemisolvate monohydrate

Crystal data

$2 \mathrm{C}_{11} \mathrm{H}_{20} \mathrm{~N}^{+} \cdot 2 \mathrm{Cl}^{-} \cdot 0.5 \mathrm{C}_{4} \mathrm{H}_{8} \mathrm{O}_{2} \cdot \mathrm{H}_{2} \mathrm{O}$

$M_{r}=465.53$

Triclinic, $P \overline{1}$

$a=6.4941$ (11) $\AA$

$b=13.491(2) \AA$

$c=15.086(3) \AA$

$\alpha=102.911(3)^{\circ}$

$\beta=91.824(3)^{\circ}$

$\gamma=101.500(3)^{\circ}$

$V=1258.5(4) \AA^{3}$

Data collection

Bruker APEXII CCD

diffractometer

$\varphi$ and $\omega$ scans

Absorption correction: multi-scan

(SADABS; Bruker, 2015)

$T_{\min }=0.655, T_{\max }=0.746$

35014 measured reflections

Refinement

Refinement on $F^{2}$

Least-squares matrix: full

$R\left[F^{2}>2 \sigma\left(F^{2}\right)\right]=0.056$

$w R\left(F^{2}\right)=0.105$

$S=1.06$

6312 reflections

303 parameters

0 restraints
$Z=2$

$F(000)=508$

$D_{\mathrm{x}}=1.229 \mathrm{Mg} \mathrm{m}^{-3}$

Mo $K \alpha$ radiation, $\lambda=0.71073 \AA$

Cell parameters from 4531 reflections

$\theta=2.3-28.0^{\circ}$

$\mu=0.28 \mathrm{~mm}^{-1}$

$T=100 \mathrm{~K}$

Needle, clear light colourless

$0.2 \times 0.05 \times 0.05 \mathrm{~mm}$

6312 independent reflections

4285 reflections with $I>2 \sigma(I)$

$R_{\text {int }}=0.096$

$\theta_{\max }=28.4^{\circ}, \theta_{\min }=1.6^{\circ}$

$h=-8 \rightarrow 8$

$k=-18 \rightarrow 17$

$l=-20 \rightarrow 20$

Hydrogen site location: mixed

$\mathrm{H}$ atoms treated by a mixture of independent and constrained refinement

$w=1 /\left[\sigma^{2}\left(F_{\mathrm{o}}^{2}\right)+(0.0415 P)^{2}+0.1332 P\right]$

where $P=\left(F_{\mathrm{o}}^{2}+2 F_{\mathrm{c}}^{2}\right) / 3$

$(\Delta / \sigma)_{\max }=0.001$

$\Delta \rho_{\max }=0.49$ e $\AA^{-3}$

$\Delta \rho_{\min }=-0.39$ e $\AA^{-3}$ 


\section{Special details}

Geometry. All esds (except the esd in the dihedral angle between two 1.s. planes) are estimated using the full covariance matrix. The cell esds are taken into account individually in the estimation of esds in distances, angles and torsion angles; correlations between esds in cell parameters are only used when they are defined by crystal symmetry. An approximate (isotropic) treatment of cell esds is used for estimating esds involving l.s. planes.

Fractional atomic coordinates and isotropic or equivalent isotropic displacement parameters $\left(\AA^{2}\right)$

\begin{tabular}{|c|c|c|c|c|}
\hline & $x$ & $y$ & $z$ & $U_{\text {iso }} * / U_{\text {eq }}$ \\
\hline Cl1 & $0.87597(8)$ & $0.25420(4)$ & $0.41712(4)$ & $0.02117(14)$ \\
\hline $\mathrm{O} 1$ & $0.7543(3)$ & $0.01392(17)$ & $0.29766(13)$ & $0.0350(4)$ \\
\hline H1D & $0.744(5)$ & $-0.019(3)$ & $0.337(2)$ & $0.067(12)^{*}$ \\
\hline $\mathrm{H} 1 \mathrm{E}$ & $0.789(5)$ & $0.081(3)$ & $0.331(2)$ & $0.069(11)^{*}$ \\
\hline N1 & $0.3859(3)$ & $0.22378(16)$ & $0.37519(13)$ & $0.0184(4)$ \\
\hline H1A & $0.347(4)$ & $0.202(2)$ & $0.4221(18)$ & $0.034(7)^{*}$ \\
\hline H1B & $0.331(4)$ & $0.280(2)$ & $0.3786(16)$ & $0.032(7)^{*}$ \\
\hline $\mathrm{H} 1 \mathrm{C}$ & $0.540(5)$ & $0.242(2)$ & $0.3820(18)$ & $0.049(8)^{*}$ \\
\hline $\mathrm{C} 1$ & $0.3307(3)$ & $0.17360(15)$ & $0.20310(13)$ & $0.0124(4)$ \\
\hline $\mathrm{C} 2$ & $0.1981(3)$ & $0.25504(15)$ & $0.19496(14)$ & $0.0149(4)$ \\
\hline $\mathrm{H} 2 \mathrm{~A}$ & 0.0482 & 0.2266 & 0.2017 & $0.018^{*}$ \\
\hline $\mathrm{H} 2 \mathrm{~B}$ & 0.2472 & 0.3185 & 0.2443 & $0.018^{*}$ \\
\hline $\mathrm{C} 3$ & $0.2196(3)$ & $0.28259(15)$ & $0.10183(14)$ & $0.0150(4)$ \\
\hline H3 & 0.1337 & 0.3354 & 0.0972 & $0.018^{*}$ \\
\hline $\mathrm{C} 4$ & $0.4520(3)$ & $0.32784(16)$ & $0.09241(14)$ & $0.0161(4)$ \\
\hline $\mathrm{H} 4 \mathrm{~A}$ & 0.4666 & 0.3470 & 0.0329 & $0.019 *$ \\
\hline H4B & 0.5031 & 0.3914 & 0.1414 & $0.019^{*}$ \\
\hline $\mathrm{C} 5$ & $0.5838(3)$ & $0.24691(15)$ & $0.09893(13)$ & $0.0141(4)$ \\
\hline H5 & 0.7351 & 0.2763 & 0.0925 & $0.017 *$ \\
\hline C6 & $0.5625(3)$ & $0.21869(16)$ & $0.19195(13)$ & $0.0131(4)$ \\
\hline H6A & 0.6486 & 0.1669 & 0.1966 & $0.016^{*}$ \\
\hline H6B & 0.6157 & 0.2816 & 0.2414 & $0.016^{*}$ \\
\hline $\mathrm{C} 7$ & $0.5040(3)$ & $0.14874(16)$ & $0.02254(14)$ & $0.0172(4)$ \\
\hline H7A & 0.5184 & 0.1662 & -0.0376 & $0.021 *$ \\
\hline H7B & 0.5894 & 0.0964 & 0.0261 & $0.021^{*}$ \\
\hline $\mathrm{C} 8$ & $0.2722(3)$ & $0.10384(16)$ & $0.03253(14)$ & $0.0160(4)$ \\
\hline H8 & 0.2205 & 0.0399 & -0.0172 & $0.019 *$ \\
\hline C9 & $0.1409(3)$ & $0.18475(16)$ & $0.02568(14)$ & $0.0174(4)$ \\
\hline H9A & 0.1532 & 0.2025 & -0.0344 & $0.021^{*}$ \\
\hline H9B & -0.0094 & 0.1558 & 0.0312 & $0.021^{*}$ \\
\hline $\mathrm{C} 10$ & $0.2512(3)$ & $0.07589(15)$ & $0.12521(13)$ & $0.0153(4)$ \\
\hline H10A & 0.3343 & 0.0228 & 0.1293 & $0.018^{*}$ \\
\hline H10B & 0.1017 & 0.0461 & 0.1314 & $0.018^{*}$ \\
\hline C11 & $0.3041(3)$ & $0.13900(16)$ & $0.29224(13)$ & $0.0166(4)$ \\
\hline H11A & 0.1525 & 0.1115 & 0.2965 & $0.020^{*}$ \\
\hline H11B & 0.3785 & 0.0815 & 0.2912 & $0.020^{*}$ \\
\hline $\mathrm{Cl} 2$ & $1.31606(7)$ & $0.11138(4)$ & $0.53791(3)$ & $0.01540(12)$ \\
\hline $\mathrm{O} 2$ & $0.4015(2)$ & $0.44325(11)$ & 0.41377 (10) & $0.0234(4)$ \\
\hline $\mathrm{C} 23$ & $0.2855(3)$ & $0.49326(18)$ & $0.48258(16)$ & $0.0282(6)$ \\
\hline
\end{tabular}




$\begin{array}{lllll}\text { H23A } & 0.1386 & 0.4529 & 0.4765 & 0.034^{*} \\ \text { H23B } & 0.2817 & 0.5637 & 0.4744 & 0.034^{*} \\ \text { C24 } & 0.6155(4) & 0.49844(19) & 0.42468(16) & 0.0307(6) \\ \text { H24A } & 0.6217 & 0.5691 & 0.4150 & 0.037^{*} \\ \text { H24B } & 0.6960 & 0.4621 & 0.3781 & 0.037^{*} \\ \text { N2 } & 0.8388(3) & 0.12390(16) & 0.56887(12) & 0.0161(4) \\ \text { H2C } & 0.785(4) & 0.062(2) & 0.5387(16) & 0.022(6)^{*} \\ \text { H2D } & 0.788(4) & 0.165(2) & 0.5421(17) & 0.031(7)^{*} \\ \text { H2E } & 0.977(4) & 0.1327(19) & 0.5613(16) & 0.033(7)^{*} \\ \text { C12 } & 0.8559(3) & 0.24495(15) & 0.72436(13) & 0.0113(4) \\ \text { C13 } & 1.0863(3) & 0.29809(15) & 0.71971(14) & 0.0145(4) \\ \text { H13A } & 1.1811 & 0.2563 & 0.7388 & 0.017^{*} \\ \text { H13B } & 1.1111 & 0.3024 & 0.6561 & 0.017^{*} \\ \text { C14 } & 1.1355(3) & 0.40791(16) & 0.78235(14) & 0.0157(4) \\ \text { H14 } & 1.2854 & 0.4421 & 0.7784 & 0.019^{*} \\ \text { C15 } & 0.9883(3) & 0.47258(16) & 0.75293(14) & 0.0172(4) \\ \text { H15A } & 1.0211 & 0.5437 & 0.7928 & 0.021^{*} \\ \text { H15B } & 1.0101 & 0.4781 & 0.6894 & 0.021^{*} \\ \text { C16 } & 0.7587(3) & 0.42114(16) & 0.75939(13) & 0.0154(4) \\ \text { H16 } & 0.6633 & 0.4637 & 0.7403 & 0.019^{*} \\ \text { C17 } & 0.7099(3) & 0.31176(15) & 0.69597(13) & 0.0131(4) \\ \text { H17A } & 0.7303 & 0.3170 & 0.6323 & 0.016^{*} \\ \text { H17B } & 0.5610 & 0.2781 & 0.6989 & 0.016^{*} \\ \text { C18 } & 0.7244(3) & 0.41275(16) & 0.85783(13) & 0.0173(4) \\ \text { H18A } & 0.7535 & 0.4831 & 0.8989 & 0.021^{*} \\ \text { H18B } & 0.5760 & 0.3791 & 0.8619 & 0.021^{*} \\ \text { C19 } & 0.8716(3) & 0.34845(16) & 0.88730(14) & 0.0167(4) \\ \text { H19 } & 0.8498 & 0.3433 & 0.9516 & 0.020^{*} \\ \text { C20 } & 1.1009(3) & 0.40095(16) & 0.88100(14) & 0.0181(5) \\ \text { H20A } & 1.1967 & 0.3603 & 0.9011 & 0.022^{*} \\ \text { H20B } & 1.1332 & 0.4716 & 0.9216 & 0.022^{*} \\ \text { C21 } & 0.8212(3) & 0.23899(16) & 0.82383(13) & 0.0148(4) \\ \text { H21A } & 0.6729 & 0.2053 & 0.8280 & 0.018^{*} \\ \text { H21B } & 0.9132 & 0.1961 & 0.8434 & 0.018^{*} \\ \text { C22 } & 0.8034(3) & 0.13331(15) & 0.66746(13) & 0.0135(4) \\ \text { H22A } & 0.8908 & 0.0923 & 0.6928 & 0.016^{*} \\ \text { H22B } & 0.6538 & 0.1027 & 0.6727 & 0.016^{*} \\ & & & & \end{array}$

Atomic displacement parameters $\left(\AA^{2}\right)$

\begin{tabular}{lllllll}
\hline & $U^{11}$ & $U^{22}$ & $U^{33}$ & $U^{12}$ & $U^{13}$ & $U^{23}$ \\
\hline C11 & $0.0178(3)$ & $0.0247(3)$ & $0.0229(3)$ & $0.0049(2)$ & $0.0017(2)$ & $0.0091(2)$ \\
O1 & $0.0399(11)$ & $0.0353(12)$ & $0.0310(11)$ & $0.0050(9)$ & $0.0045(9)$ & $0.0126(10)$ \\
N1 & $0.0183(10)$ & $0.0238(11)$ & $0.0151(10)$ & $0.0056(9)$ & $0.0019(8)$ & $0.0071(8)$ \\
C1 & $0.0111(9)$ & $0.0120(10)$ & $0.0140(10)$ & $0.0014(8)$ & $0.0006(8)$ & $0.0037(8)$ \\
C2 & $0.0119(10)$ & $0.0136(10)$ & $0.0197(11)$ & $0.0041(8)$ & $0.0023(8)$ & $0.0035(9)$ \\
C3 & $0.0145(10)$ & $0.0123(10)$ & $0.0194(11)$ & $0.0052(8)$ & $-0.0013(8)$ & $0.0043(8)$ \\
C4 & $0.0187(11)$ & $0.0146(11)$ & $0.0162(11)$ & $0.0026(9)$ & $-0.0006(9)$ & $0.0070(9)$
\end{tabular}




$\begin{array}{lllllll}\text { C5 } & 0.0100(10) & 0.0165(11) & 0.0152(10) & 0.0008(8) & 0.0025(8) & 0.0045(8) \\ \text { C6 } & 0.0111(10) & 0.0144(10) & 0.0138(10) & 0.0029(8) & -0.0010(8) & 0.0034(8) \\ \text { C7 } & 0.0189(11) & 0.0192(11) & 0.0150(11) & 0.0079(9) & 0.0034(9) & 0.0037(9) \\ \text { C8 } & 0.0179(11) & 0.0142(11) & 0.0137(10) & 0.0027(9) & -0.0021(8) & -0.0002(8) \\ \text { C9 } & 0.0146(10) & 0.0188(11) & 0.0179(11) & 0.0013(9) & -0.0054(8) & 0.0052(9) \\ \text { C10 } & 0.0143(10) & 0.0107(10) & 0.0204(11) & 0.0019(8) & -0.0006(8) & 0.0036(8) \\ \text { C11 } & 0.0140(10) & 0.0172(11) & 0.0183(11) & 0.0007(9) & 0.0006(8) & 0.0061(9) \\ \text { C12 } & 0.0146(2) & 0.0168(3) & 0.0135(2) & 0.0022(2) & -0.00028(19) & 0.00194(19) \\ \text { O2 } & 0.0235(8) & 0.0231(9) & 0.0205(8) & 0.0020(7) & -0.0008(7) & 0.0018(7) \\ \text { C23 } & 0.0159(11) & 0.0221(13) & 0.0420(15) & 0.0058(10) & 0.0027(10) & -0.0034(11) \\ \text { C24 } & 0.0343(14) & 0.0258(13) & 0.0270(13) & -0.0027(11) & 0.0181(11) & 0.0018(10) \\ \text { N2 } & 0.0175(10) & 0.0147(10) & 0.0136(9) & 0.0033(8) & -0.0004(8) & -0.0013(8) \\ \text { C12 } & 0.0111(9) & 0.0121(10) & 0.0102(9) & 0.0019(8) & -0.0001(8) & 0.0019(8) \\ \text { C13 } & 0.0103(10) & 0.0169(11) & 0.0155(10) & 0.0023(8) & 0.0013(8) & 0.0025(8) \\ \text { C14 } & 0.0114(10) & 0.0133(10) & 0.0189(11) & -0.0015(8) & 0.0018(8) & 0.0001(8) \\ \text { C15 } & 0.0226(11) & 0.0119(11) & 0.0163(11) & 0.0024(9) & 0.0033(9) & 0.0026(8) \\ \text { C16 } & 0.0157(10) & 0.0163(11) & 0.0155(11) & 0.0057(9) & 0.0012(8) & 0.0040(8) \\ \text { C17 } & 0.0117(10) & 0.0170(11) & 0.0115(10) & 0.0044(8) & -0.0002(8) & 0.0042(8) \\ \text { C18 } & 0.0173(11) & 0.0182(11) & 0.0153(11) & 0.0041(9) & 0.0042(8) & 0.0010(9) \\ \text { C19 } & 0.0195(11) & 0.0201(11) & 0.0103(10) & 0.0038(9) & 0.0023(8) & 0.0033(8) \\ \text { C20 } & 0.0186(11) & 0.0169(11) & 0.0158(11) & 0.0019(9) & -0.0032(9) & 0.0000(9) \\ \text { C21 } & 0.0152(10) & 0.0172(11) & 0.0130(10) & 0.0022(9) & 0.0013(8) & 0.0065(8) \\ \text { C22 } & 0.0133(10) & 0.0129(10) & 0.0139(10) & 0.0013(8) & 0.0013(8) & 0.0039(8) \\ & & & & & & \end{array}$

Geometric parameters (A, $\stackrel{\circ}{)}$

\begin{tabular}{|c|c|c|c|}
\hline $\mathrm{O} 1-\mathrm{H} 1 \mathrm{D}$ & $0.82(3)$ & $\mathrm{C} 23-\mathrm{H} 23 \mathrm{~B}$ & 0.9900 \\
\hline $\mathrm{O} 1-\mathrm{H} 1 \mathrm{E}$ & $0.91(4)$ & $\mathrm{C} 23-\mathrm{C} 24^{\mathrm{i}}$ & $1.493(3)$ \\
\hline $\mathrm{N} 1-\mathrm{H} 1 \mathrm{~A}$ & $0.85(3)$ & $\mathrm{C} 24-\mathrm{C} 23^{\mathrm{i}}$ & $1.493(3)$ \\
\hline $\mathrm{N} 1-\mathrm{H} 1 \mathrm{~B}$ & $0.89(3)$ & $\mathrm{C} 24-\mathrm{H} 24 \mathrm{~A}$ & 0.9900 \\
\hline $\mathrm{N} 1-\mathrm{H} 1 \mathrm{C}$ & $0.98(3)$ & $\mathrm{C} 24-\mathrm{H} 24 \mathrm{~B}$ & 0.9900 \\
\hline $\mathrm{N} 1-\mathrm{C} 11$ & $1.494(3)$ & $\mathrm{N} 2-\mathrm{H} 2 \mathrm{C}$ & $0.86(2)$ \\
\hline $\mathrm{C} 1-\mathrm{C} 2$ & $1.547(3)$ & $\mathrm{N} 2-\mathrm{H} 2 \mathrm{D}$ & $0.86(3)$ \\
\hline $\mathrm{C} 1-\mathrm{C} 6$ & $1.537(3)$ & $\mathrm{N} 2-\mathrm{H} 2 \mathrm{E}$ & $0.90(3)$ \\
\hline $\mathrm{C} 1-\mathrm{C} 10$ & $1.544(3)$ & $\mathrm{N} 2-\mathrm{C} 22$ & $1.493(3)$ \\
\hline $\mathrm{C} 1-\mathrm{C} 11$ & $1.523(3)$ & $\mathrm{C} 12-\mathrm{C} 13$ & $1.536(3)$ \\
\hline $\mathrm{C} 2-\mathrm{H} 2 \mathrm{~A}$ & 0.9900 & $\mathrm{C} 12-\mathrm{C} 17$ & $1.543(3)$ \\
\hline $\mathrm{C} 2-\mathrm{H} 2 \mathrm{~B}$ & 0.9900 & $\mathrm{C} 12-\mathrm{C} 21$ & $1.542(3)$ \\
\hline $\mathrm{C} 2-\mathrm{C} 3$ & $1.535(3)$ & $\mathrm{C} 12-\mathrm{C} 22$ & $1.523(3)$ \\
\hline $\mathrm{C} 3-\mathrm{H} 3$ & 1.0000 & $\mathrm{C} 13-\mathrm{H} 13 \mathrm{~A}$ & 0.9900 \\
\hline $\mathrm{C} 3-\mathrm{C} 4$ & $1.535(3)$ & $\mathrm{C} 13-\mathrm{H} 13 \mathrm{~B}$ & 0.9900 \\
\hline $\mathrm{C} 3-\mathrm{C} 9$ & $1.529(3)$ & $\mathrm{C} 13-\mathrm{C} 14$ & $1.535(3)$ \\
\hline $\mathrm{C} 4-\mathrm{H} 4 \mathrm{~A}$ & 0.9900 & C14-H14 & 1.0000 \\
\hline $\mathrm{C} 4-\mathrm{H} 4 \mathrm{~B}$ & 0.9900 & $\mathrm{C} 14-\mathrm{C} 15$ & $1.533(3)$ \\
\hline $\mathrm{C} 4-\mathrm{C} 5$ & $1.533(3)$ & $\mathrm{C} 14-\mathrm{C} 20$ & $1.533(3)$ \\
\hline $\mathrm{C} 5-\mathrm{H} 5$ & 1.0000 & $\mathrm{C} 15-\mathrm{H} 15 \mathrm{~A}$ & 0.9900 \\
\hline $\mathrm{C} 5-\mathrm{C} 6$ & $1.537(3)$ & $\mathrm{C} 15-\mathrm{H} 15 \mathrm{~B}$ & 0.9900 \\
\hline $\mathrm{C} 5-\mathrm{C} 7$ & $1.535(3)$ & $\mathrm{C} 15-\mathrm{C} 16$ & $1.530(3)$ \\
\hline
\end{tabular}




\begin{tabular}{|c|c|c|c|}
\hline C6-H6A & 0.9900 & $\mathrm{C} 16-\mathrm{H} 16$ & 1.0000 \\
\hline C6-H6B & 0.9900 & $\mathrm{C} 16-\mathrm{C} 17$ & $1.535(3)$ \\
\hline C7-H7A & 0.9900 & $\mathrm{C} 16-\mathrm{C} 18$ & $1.534(3)$ \\
\hline C7-H7B & 0.9900 & C17-H17A & 0.9900 \\
\hline $\mathrm{C} 7-\mathrm{C} 8$ & $1.532(3)$ & C17-H17B & 0.9900 \\
\hline $\mathrm{C} 8-\mathrm{H} 8$ & 1.0000 & $\mathrm{C} 18-\mathrm{H} 18 \mathrm{~A}$ & 0.9900 \\
\hline $\mathrm{C} 8-\mathrm{C} 9$ & $1.532(3)$ & $\mathrm{C} 18-\mathrm{H} 18 \mathrm{~B}$ & 0.9900 \\
\hline $\mathrm{C} 8-\mathrm{C} 10$ & $1.531(3)$ & $\mathrm{C} 18-\mathrm{C} 19$ & $1.530(3)$ \\
\hline C9-H9A & 0.9900 & $\mathrm{C} 19-\mathrm{H} 19$ & 1.0000 \\
\hline C9-H9B & 0.9900 & $\mathrm{C} 19-\mathrm{C} 20$ & $1.531(3)$ \\
\hline $\mathrm{C} 10-\mathrm{H} 10 \mathrm{~A}$ & 0.9900 & $\mathrm{C} 19-\mathrm{C} 21$ & $1.536(3)$ \\
\hline $\mathrm{C} 10-\mathrm{H} 10 \mathrm{~B}$ & 0.9900 & $\mathrm{C} 20-\mathrm{H} 20 \mathrm{~A}$ & 0.9900 \\
\hline $\mathrm{C} 11-\mathrm{H} 11 \mathrm{~A}$ & 0.9900 & $\mathrm{C} 20-\mathrm{H} 20 \mathrm{~B}$ & 0.9900 \\
\hline $\mathrm{C} 11-\mathrm{H} 11 \mathrm{~B}$ & 0.9900 & $\mathrm{C} 21-\mathrm{H} 21 \mathrm{~A}$ & 0.9900 \\
\hline $\mathrm{O} 2-\mathrm{C} 23$ & $1.429(3)$ & $\mathrm{C} 21-\mathrm{H} 21 \mathrm{~B}$ & 0.9900 \\
\hline $\mathrm{O} 2-\mathrm{C} 24$ & $1.425(3)$ & $\mathrm{C} 22-\mathrm{H} 22 \mathrm{~A}$ & 0.9900 \\
\hline $\mathrm{C} 23-\mathrm{H} 23 \mathrm{~A}$ & 0.9900 & $\mathrm{C} 22-\mathrm{H} 22 \mathrm{~B}$ & 0.9900 \\
\hline $\mathrm{H} 1 \mathrm{D}-\mathrm{O} 1-\mathrm{H} 1 \mathrm{E}$ & $102(3)$ & $\mathrm{C} 24-\mathrm{C} 23-\mathrm{H} 23 \mathrm{~B}$ & 109.5 \\
\hline $\mathrm{H} 1 \mathrm{~A}-\mathrm{N} 1-\mathrm{H} 1 \mathrm{~B}$ & $105(2)$ & $\mathrm{O} 2-\mathrm{C} 24-\mathrm{C} 23^{\mathrm{i}}$ & $111.48(18)$ \\
\hline $\mathrm{H} 1 \mathrm{~A}-\mathrm{N} 1-\mathrm{H} 1 \mathrm{C}$ & $106(2)$ & $\mathrm{O} 2-\mathrm{C} 24-\mathrm{H} 24 \mathrm{~A}$ & 109.3 \\
\hline $\mathrm{H} 1 \mathrm{~B}-\mathrm{N} 1-\mathrm{H} 1 \mathrm{C}$ & $111(2)$ & $\mathrm{O} 2-\mathrm{C} 24-\mathrm{H} 24 \mathrm{~B}$ & 109.3 \\
\hline $\mathrm{C} 11-\mathrm{N} 1-\mathrm{H} 1 \mathrm{~A}$ & $108.3(17)$ & $\mathrm{C} 23^{\mathrm{i}}-\mathrm{C} 24-\mathrm{H} 24 \mathrm{~A}$ & 109.3 \\
\hline $\mathrm{C} 11-\mathrm{N} 1-\mathrm{H} 1 \mathrm{~B}$ & $113.6(16)$ & $\mathrm{C} 23^{\mathrm{i}}-\mathrm{C} 24-\mathrm{H} 24 \mathrm{~B}$ & 109.3 \\
\hline $\mathrm{C} 11-\mathrm{N} 1-\mathrm{H} 1 \mathrm{C}$ & $112.3(16)$ & $\mathrm{H} 24 \mathrm{~A}-\mathrm{C} 24-\mathrm{H} 24 \mathrm{~B}$ & 108.0 \\
\hline $\mathrm{C} 6-\mathrm{C} 1-\mathrm{C} 2$ & $108.98(16)$ & $\mathrm{H} 2 \mathrm{C}-\mathrm{N} 2-\mathrm{H} 2 \mathrm{D}$ & $106(2)$ \\
\hline $\mathrm{C} 6-\mathrm{C} 1-\mathrm{C} 10$ & $108.68(16)$ & $\mathrm{H} 2 \mathrm{C}-\mathrm{N} 2-\mathrm{H} 2 \mathrm{E}$ & $105(2)$ \\
\hline $\mathrm{C} 10-\mathrm{C} 1-\mathrm{C} 2$ & $108.41(16)$ & $\mathrm{H} 2 \mathrm{D}-\mathrm{N} 2-\mathrm{H} 2 \mathrm{E}$ & $108(2)$ \\
\hline $\mathrm{C} 11-\mathrm{C} 1-\mathrm{C} 2$ & $111.88(16)$ & $\mathrm{C} 22-\mathrm{N} 2-\mathrm{H} 2 \mathrm{C}$ & $109.5(15)$ \\
\hline $\mathrm{C} 11-\mathrm{C} 1-\mathrm{C} 6$ & $111.96(16)$ & $\mathrm{C} 22-\mathrm{N} 2-\mathrm{H} 2 \mathrm{D}$ & $116.8(16)$ \\
\hline $\mathrm{C} 11-\mathrm{C} 1-\mathrm{C} 10$ & $106.81(16)$ & $\mathrm{C} 22-\mathrm{N} 2-\mathrm{H} 2 \mathrm{E}$ & $110.1(16)$ \\
\hline $\mathrm{C} 1-\mathrm{C} 2-\mathrm{H} 2 \mathrm{~A}$ & 109.7 & $\mathrm{C} 13-\mathrm{C} 12-\mathrm{C} 17$ & $109.06(16)$ \\
\hline $\mathrm{C} 1-\mathrm{C} 2-\mathrm{H} 2 \mathrm{~B}$ & 109.7 & $\mathrm{C} 13-\mathrm{C} 12-\mathrm{C} 21$ & $108.64(16)$ \\
\hline $\mathrm{H} 2 \mathrm{~A}-\mathrm{C} 2-\mathrm{H} 2 \mathrm{~B}$ & 108.2 & $\mathrm{C} 21-\mathrm{C} 12-\mathrm{C} 17$ & $108.24(15)$ \\
\hline $\mathrm{C} 3-\mathrm{C} 2-\mathrm{C} 1$ & $109.98(15)$ & $\mathrm{C} 22-\mathrm{C} 12-\mathrm{C} 13$ & $112.02(15)$ \\
\hline $\mathrm{C} 3-\mathrm{C} 2-\mathrm{H} 2 \mathrm{~A}$ & 109.7 & $\mathrm{C} 22-\mathrm{C} 12-\mathrm{C} 17$ & $112.31(16)$ \\
\hline $\mathrm{C} 3-\mathrm{C} 2-\mathrm{H} 2 \mathrm{~B}$ & 109.7 & $\mathrm{C} 22-\mathrm{C} 12-\mathrm{C} 21$ & $106.42(15)$ \\
\hline $\mathrm{C} 2-\mathrm{C} 3-\mathrm{H} 3$ & 109.4 & $\mathrm{C} 12-\mathrm{C} 13-\mathrm{H} 13 \mathrm{~A}$ & 109.6 \\
\hline $\mathrm{C} 4-\mathrm{C} 3-\mathrm{C} 2$ & $109.32(16)$ & $\mathrm{C} 12-\mathrm{C} 13-\mathrm{H} 13 \mathrm{~B}$ & 109.6 \\
\hline $\mathrm{C} 4-\mathrm{C} 3-\mathrm{H} 3$ & 109.4 & $\mathrm{H} 13 \mathrm{~A}-\mathrm{C} 13-\mathrm{H} 13 \mathrm{~B}$ & 108.1 \\
\hline $\mathrm{C} 9-\mathrm{C} 3-\mathrm{C} 2$ & $109.66(16)$ & $\mathrm{C} 14-\mathrm{C} 13-\mathrm{C} 12$ & $110.21(15)$ \\
\hline $\mathrm{C} 9-\mathrm{C} 3-\mathrm{H} 3$ & 109.4 & $\mathrm{C} 14-\mathrm{C} 13-\mathrm{H} 13 \mathrm{~A}$ & 109.6 \\
\hline $\mathrm{C} 9-\mathrm{C} 3-\mathrm{C} 4$ & $109.58(16)$ & $\mathrm{C} 14-\mathrm{C} 13-\mathrm{H} 13 \mathrm{~B}$ & 109.6 \\
\hline $\mathrm{C} 3-\mathrm{C} 4-\mathrm{H} 4 \mathrm{~A}$ & 109.8 & $\mathrm{C} 13-\mathrm{C} 14-\mathrm{H} 14$ & 109.6 \\
\hline $\mathrm{C} 3-\mathrm{C} 4-\mathrm{H} 4 \mathrm{~B}$ & 109.8 & $\mathrm{C} 15-\mathrm{C} 14-\mathrm{C} 13$ & $109.61(16)$ \\
\hline $\mathrm{H} 4 \mathrm{~A}-\mathrm{C} 4-\mathrm{H} 4 \mathrm{~B}$ & 108.2 & $\mathrm{C} 15-\mathrm{C} 14-\mathrm{H} 14$ & 109.6 \\
\hline $\mathrm{C} 5-\mathrm{C} 4-\mathrm{C} 3$ & $109.55(16)$ & $\mathrm{C} 20-\mathrm{C} 14-\mathrm{C} 13$ & $109.53(17)$ \\
\hline $\mathrm{C} 5-\mathrm{C} 4-\mathrm{H} 4 \mathrm{~A}$ & 109.8 & $\mathrm{C} 20-\mathrm{C} 14-\mathrm{H} 14$ & 109.6 \\
\hline
\end{tabular}




\begin{tabular}{|c|c|c|c|}
\hline $\mathrm{C} 5-\mathrm{C} 4-\mathrm{H} 4 \mathrm{~B}$ & 109.8 & $\mathrm{C} 20-\mathrm{C} 14-\mathrm{C} 15$ & $108.95(16)$ \\
\hline $\mathrm{C} 4-\mathrm{C} 5-\mathrm{H} 5$ & 109.5 & $\mathrm{C} 14-\mathrm{C} 15-\mathrm{H} 15 \mathrm{~A}$ & 109.7 \\
\hline $\mathrm{C} 4-\mathrm{C} 5-\mathrm{C} 6$ & $109.41(15)$ & $\mathrm{C} 14-\mathrm{C} 15-\mathrm{H} 15 \mathrm{~B}$ & 109.7 \\
\hline $\mathrm{C} 4-\mathrm{C} 5-\mathrm{C} 7$ & $109.42(16)$ & $\mathrm{H} 15 \mathrm{~A}-\mathrm{C} 15-\mathrm{H} 15 \mathrm{~B}$ & 108.2 \\
\hline $\mathrm{C} 6-\mathrm{C} 5-\mathrm{H} 5$ & 109.5 & $\mathrm{C} 16-\mathrm{C} 15-\mathrm{C} 14$ & $109.89(16)$ \\
\hline $\mathrm{C} 7-\mathrm{C} 5-\mathrm{H} 5$ & 109.5 & $\mathrm{C} 16-\mathrm{C} 15-\mathrm{H} 15 \mathrm{~A}$ & 109.7 \\
\hline $\mathrm{C} 7-\mathrm{C} 5-\mathrm{C} 6$ & $109.35(16)$ & $\mathrm{C} 16-\mathrm{C} 15-\mathrm{H} 15 \mathrm{~B}$ & 109.7 \\
\hline $\mathrm{C} 1-\mathrm{C} 6-\mathrm{C} 5$ & $110.25(16)$ & $\mathrm{C} 15-\mathrm{C} 16-\mathrm{H} 16$ & 109.6 \\
\hline $\mathrm{C} 1-\mathrm{C} 6-\mathrm{H} 6 \mathrm{~A}$ & 109.6 & $\mathrm{C} 15-\mathrm{C} 16-\mathrm{C} 17$ & $108.88(16)$ \\
\hline $\mathrm{C} 1-\mathrm{C} 6-\mathrm{H} 6 \mathrm{~B}$ & 109.6 & $\mathrm{C} 15-\mathrm{C} 16-\mathrm{C} 18$ & $109.79(17)$ \\
\hline $\mathrm{C} 5-\mathrm{C} 6-\mathrm{H} 6 \mathrm{~A}$ & 109.6 & $\mathrm{C} 17-\mathrm{C} 16-\mathrm{H} 16$ & 109.6 \\
\hline $\mathrm{C} 5-\mathrm{C} 6-\mathrm{H} 6 \mathrm{~B}$ & 109.6 & $\mathrm{C} 18-\mathrm{C} 16-\mathrm{H} 16$ & 109.6 \\
\hline $\mathrm{H} 6 \mathrm{~A}-\mathrm{C} 6-\mathrm{H} 6 \mathrm{~B}$ & 108.1 & $\mathrm{C} 18-\mathrm{C} 16-\mathrm{C} 17$ & $109.39(16)$ \\
\hline $\mathrm{C} 5-\mathrm{C} 7-\mathrm{H} 7 \mathrm{~A}$ & 109.8 & $\mathrm{C} 12-\mathrm{C} 17-\mathrm{H} 17 \mathrm{~A}$ & 109.6 \\
\hline $\mathrm{C} 5-\mathrm{C} 7-\mathrm{H} 7 \mathrm{~B}$ & 109.8 & $\mathrm{C} 12-\mathrm{C} 17-\mathrm{H} 17 \mathrm{~B}$ & 109.6 \\
\hline $\mathrm{H} 7 \mathrm{~A}-\mathrm{C} 7-\mathrm{H} 7 \mathrm{~B}$ & 108.2 & $\mathrm{C} 16-\mathrm{C} 17-\mathrm{C} 12$ & $110.35(16)$ \\
\hline $\mathrm{C} 8-\mathrm{C} 7-\mathrm{C} 5$ & $109.49(16)$ & $\mathrm{C} 16-\mathrm{C} 17-\mathrm{H} 17 \mathrm{~A}$ & 109.6 \\
\hline $\mathrm{C} 8-\mathrm{C} 7-\mathrm{H} 7 \mathrm{~A}$ & 109.8 & $\mathrm{C} 16-\mathrm{C} 17-\mathrm{H} 17 \mathrm{~B}$ & 109.6 \\
\hline $\mathrm{C} 8-\mathrm{C} 7-\mathrm{H} 7 \mathrm{~B}$ & 109.8 & $\mathrm{H} 17 \mathrm{~A}-\mathrm{C} 17-\mathrm{H} 17 \mathrm{~B}$ & 108.1 \\
\hline $\mathrm{C} 7-\mathrm{C} 8-\mathrm{H} 8$ & 109.4 & $\mathrm{C} 16-\mathrm{C} 18-\mathrm{H} 18 \mathrm{~A}$ & 109.8 \\
\hline $\mathrm{C} 9-\mathrm{C} 8-\mathrm{C} 7$ & $109.42(17)$ & $\mathrm{C} 16-\mathrm{C} 18-\mathrm{H} 18 \mathrm{~B}$ & 109.8 \\
\hline $\mathrm{C} 9-\mathrm{C} 8-\mathrm{H} 8$ & 109.4 & $\mathrm{H} 18 \mathrm{~A}-\mathrm{C} 18-\mathrm{H} 18 \mathrm{~B}$ & 108.2 \\
\hline $\mathrm{C} 10-\mathrm{C} 8-\mathrm{C} 7$ & $109.55(16)$ & $\mathrm{C} 19-\mathrm{C} 18-\mathrm{C} 16$ & $109.57(16)$ \\
\hline $\mathrm{C} 10-\mathrm{C} 8-\mathrm{H} 8$ & 109.4 & $\mathrm{C} 19-\mathrm{C} 18-\mathrm{H} 18 \mathrm{~A}$ & 109.8 \\
\hline $\mathrm{C} 10-\mathrm{C} 8-\mathrm{C} 9$ & $109.59(16)$ & $\mathrm{C} 19-\mathrm{C} 18-\mathrm{H} 18 \mathrm{~B}$ & 109.8 \\
\hline $\mathrm{C} 3-\mathrm{C} 9-\mathrm{C} 8$ & $109.52(16)$ & $\mathrm{C} 18-\mathrm{C} 19-\mathrm{H} 19$ & 109.6 \\
\hline $\mathrm{C} 3-\mathrm{C} 9-\mathrm{H} 9 \mathrm{~A}$ & 109.8 & $\mathrm{C} 18-\mathrm{C} 19-\mathrm{C} 20$ & $109.50(17)$ \\
\hline С $3-\mathrm{C} 9-\mathrm{H} 9 \mathrm{~B}$ & 109.8 & $\mathrm{C} 18-\mathrm{C} 19-\mathrm{C} 21$ & $108.92(17)$ \\
\hline $\mathrm{C} 8-\mathrm{C} 9-\mathrm{H} 9 \mathrm{~A}$ & 109.8 & $\mathrm{C} 20-\mathrm{C} 19-\mathrm{H} 19$ & 109.6 \\
\hline $\mathrm{C} 8-\mathrm{C} 9-\mathrm{H} 9 \mathrm{~B}$ & 109.8 & $\mathrm{C} 20-\mathrm{C} 19-\mathrm{C} 21$ & $109.70(16)$ \\
\hline $\mathrm{H} 9 \mathrm{~A}-\mathrm{C} 9-\mathrm{H} 9 \mathrm{~B}$ & 108.2 & $\mathrm{C} 21-\mathrm{C} 19-\mathrm{H} 19$ & 109.6 \\
\hline $\mathrm{C} 1-\mathrm{C} 10-\mathrm{H} 10 \mathrm{~A}$ & 109.6 & $\mathrm{C} 14-\mathrm{C} 20-\mathrm{H} 20 \mathrm{~A}$ & 109.7 \\
\hline $\mathrm{C} 1-\mathrm{C} 10-\mathrm{H} 10 \mathrm{~B}$ & 109.6 & $\mathrm{C} 14-\mathrm{C} 20-\mathrm{H} 20 \mathrm{~B}$ & 109.7 \\
\hline $\mathrm{C} 8-\mathrm{C} 10-\mathrm{C} 1$ & $110.29(16)$ & $\mathrm{C} 19-\mathrm{C} 20-\mathrm{C} 14$ & $109.72(16)$ \\
\hline $\mathrm{C} 8-\mathrm{C} 10-\mathrm{H} 10 \mathrm{~A}$ & 109.6 & $\mathrm{C} 19-\mathrm{C} 20-\mathrm{H} 20 \mathrm{~A}$ & 109.7 \\
\hline $\mathrm{C} 8-\mathrm{C} 10-\mathrm{H} 10 \mathrm{~B}$ & 109.6 & $\mathrm{C} 19-\mathrm{C} 20-\mathrm{H} 20 \mathrm{~B}$ & 109.7 \\
\hline $\mathrm{H} 10 \mathrm{~A}-\mathrm{C} 10-\mathrm{H} 10 \mathrm{~B}$ & 108.1 & $\mathrm{H} 20 \mathrm{~A}-\mathrm{C} 20-\mathrm{H} 20 \mathrm{~B}$ & 108.2 \\
\hline $\mathrm{N} 1-\mathrm{C} 11-\mathrm{C} 1$ & $113.76(17)$ & $\mathrm{C} 12-\mathrm{C} 21-\mathrm{H} 21 \mathrm{~A}$ & 109.6 \\
\hline $\mathrm{N} 1-\mathrm{C} 11-\mathrm{H} 11 \mathrm{~A}$ & 108.8 & $\mathrm{C} 12-\mathrm{C} 21-\mathrm{H} 21 \mathrm{~B}$ & 109.6 \\
\hline $\mathrm{N} 1-\mathrm{C} 11-\mathrm{H} 11 \mathrm{~B}$ & 108.8 & $\mathrm{C} 19-\mathrm{C} 21-\mathrm{C} 12$ & $110.50(16)$ \\
\hline $\mathrm{C} 1-\mathrm{C} 11-\mathrm{H} 11 \mathrm{~A}$ & 108.8 & $\mathrm{C} 19-\mathrm{C} 21-\mathrm{H} 21 \mathrm{~A}$ & 109.6 \\
\hline $\mathrm{C} 1-\mathrm{C} 11-\mathrm{H} 11 \mathrm{~B}$ & 108.8 & $\mathrm{C} 19-\mathrm{C} 21-\mathrm{H} 21 \mathrm{~B}$ & 109.6 \\
\hline $\mathrm{H} 11 \mathrm{~A}-\mathrm{C} 11-\mathrm{H} 11 \mathrm{~B}$ & 107.7 & $\mathrm{H} 21 \mathrm{~A}-\mathrm{C} 21-\mathrm{H} 21 \mathrm{~B}$ & 108.1 \\
\hline $\mathrm{C} 24-\mathrm{O} 2-\mathrm{C} 23$ & $109.78(16)$ & $\mathrm{N} 2-\mathrm{C} 22-\mathrm{C} 12$ & $113.72(16)$ \\
\hline $\mathrm{O} 2-\mathrm{C} 23-\mathrm{H} 23 \mathrm{~A}$ & 109.5 & $\mathrm{~N} 2-\mathrm{C} 22-\mathrm{H} 22 \mathrm{~A}$ & 108.8 \\
\hline $\mathrm{O} 2-\mathrm{C} 23-\mathrm{H} 23 \mathrm{~B}$ & 109.5 & $\mathrm{~N} 2-\mathrm{C} 22-\mathrm{H} 22 \mathrm{~B}$ & 108.8 \\
\hline $\mathrm{O} 2-\mathrm{C} 23-\mathrm{C} 24^{\mathrm{i}}$ & $110.56(18)$ & $\mathrm{C} 12-\mathrm{C} 22-\mathrm{H} 22 \mathrm{~A}$ & 108.8 \\
\hline $\mathrm{H} 23 \mathrm{~A}-\mathrm{C} 23-\mathrm{H} 23 \mathrm{~B}$ & 108.1 & $\mathrm{C} 12-\mathrm{C} 22-\mathrm{H} 22 \mathrm{~B}$ & 108.8 \\
\hline
\end{tabular}




$\mathrm{C} 24-\mathrm{C} 23-\mathrm{H} 23 \mathrm{~A}$
$\mathrm{C} 1-\mathrm{C} 2-\mathrm{C} 3-\mathrm{C} 4$
$\mathrm{C} 1-\mathrm{C} 2-\mathrm{C} 3-\mathrm{C} 9$
$\mathrm{C} 2-\mathrm{C} 1-\mathrm{C} 6-\mathrm{C} 5$
$\mathrm{C} 2-\mathrm{C} 1-\mathrm{C} 10-\mathrm{C} 8$
$\mathrm{C} 2-\mathrm{C} 1-\mathrm{C} 11-\mathrm{N} 1$
$\mathrm{C} 2-\mathrm{C} 3-\mathrm{C} 4-\mathrm{C} 5$
$\mathrm{C} 2-\mathrm{C} 3-\mathrm{C} 9-\mathrm{C} 8$
$\mathrm{C} 3-\mathrm{C} 4-\mathrm{C} 5-\mathrm{C} 6$
$\mathrm{C} 3-\mathrm{C} 4-\mathrm{C} 5-\mathrm{C} 7$
$\mathrm{C} 4-\mathrm{C} 3-\mathrm{C} 9-\mathrm{C} 8$
$\mathrm{C} 4-\mathrm{C} 5-\mathrm{C} 6-\mathrm{C} 1$
$\mathrm{C} 4-\mathrm{C} 5-\mathrm{C} 7-\mathrm{C} 8$
$\mathrm{C} 5-\mathrm{C} 7-\mathrm{C} 8-\mathrm{C} 9$
$\mathrm{C} 5-\mathrm{C} 7-\mathrm{C} 8-\mathrm{C} 10$
$\mathrm{C} 6-\mathrm{C} 1-\mathrm{C} 2-\mathrm{C} 3$
$\mathrm{C} 6-\mathrm{C} 1-\mathrm{C} 10-\mathrm{C} 8$
$\mathrm{C} 6-\mathrm{C} 1-\mathrm{C} 11-\mathrm{N} 1$
$\mathrm{C} 6-\mathrm{C} 5-\mathrm{C} 7-\mathrm{C} 8$
$\mathrm{C} 7-\mathrm{C} 5-\mathrm{C} 6-\mathrm{C} 1$
$\mathrm{C} 7-\mathrm{C} 8-\mathrm{C} 9-\mathrm{C} 3$
$\mathrm{C} 7-\mathrm{C} 8-\mathrm{C} 10-\mathrm{C} 1$
$\mathrm{C} 9-\mathrm{C} 3-\mathrm{C} 4-\mathrm{C} 5$
$\mathrm{C} 9-\mathrm{C} 8-\mathrm{C} 10-\mathrm{C} 1$
$\mathrm{C} 10-\mathrm{C} 1-\mathrm{C} 2-\mathrm{C} 3$
$\mathrm{C} 10-\mathrm{C} 1-\mathrm{C} 6-\mathrm{C} 5$
$\mathrm{C} 10-\mathrm{C} 1-\mathrm{C} 11-\mathrm{N} 1$
$\mathrm{C} 10-\mathrm{C} 8-\mathrm{C} 9-\mathrm{C} 3$
$\mathrm{C} 11-\mathrm{C} 1-\mathrm{C} 2-\mathrm{C} 3$
$\mathrm{C} 11-\mathrm{C} 1-\mathrm{C} 6-\mathrm{C} 5$
$\mathrm{C} 11-\mathrm{C} 1-\mathrm{C} 10-\mathrm{C} 8$
$\mathrm{C} 23-\mathrm{O} 2-\mathrm{C} 24-\mathrm{C} 23$

109.5

$-60.0(2)$

$60.1(2)$

$-58.9(2)$

$59.2(2)$

$-64.9(2)$

$60.5(2)$

$-60.0(2)$

$-60.2(2)$

$59.6(2)$

60.0 (2)

$59.8(2)$

$-59.9(2)$

$60.2(2)$

$-60.0(2)$

$59.0(2)$

$-59.1(2)$

$57.8(2)$

$59.9(2)$

$-60.0(2)$

$-60.2(2)$

$59.9(2)$

$-59.7(2)$

$-60.1(2)$

$-59.1(2)$

$59.1(2)$

$176.65(16)$

$59.9(2)$

$-176.61(16)$

$176.84(16)$

$179.95(15)$

$57.1(3)$
$\mathrm{H} 22 \mathrm{~A}-\mathrm{C} 22-\mathrm{H} 22 \mathrm{~B}$

$\mathrm{C} 24-\mathrm{O} 2-\mathrm{C} 23-\mathrm{C} 24^{\mathrm{i}}$

$\mathrm{C} 12-\mathrm{C} 13-\mathrm{C} 14-\mathrm{C} 15$

$\mathrm{C} 12-\mathrm{C} 13-\mathrm{C} 14-\mathrm{C} 20$

$\mathrm{C} 13-\mathrm{C} 12-\mathrm{C} 17-\mathrm{C} 16$

$\mathrm{C} 13-\mathrm{C} 12-\mathrm{C} 21-\mathrm{C} 19$

$\mathrm{C} 13-\mathrm{C} 12-\mathrm{C} 22-\mathrm{N} 2$

$\mathrm{C} 13-\mathrm{C} 14-\mathrm{C} 15-\mathrm{C} 16$

$\mathrm{C} 13-\mathrm{C} 14-\mathrm{C} 20-\mathrm{C} 19$

$\mathrm{C} 14-\mathrm{C} 15-\mathrm{C} 16-\mathrm{C} 17$

$\mathrm{C} 14-\mathrm{C} 15-\mathrm{C} 16-\mathrm{C} 18$

$\mathrm{C} 15-\mathrm{C} 14-\mathrm{C} 20-\mathrm{C} 19$

$\mathrm{C} 15-\mathrm{C} 16-\mathrm{C} 17-\mathrm{C} 12$

$\mathrm{C} 15-\mathrm{C} 16-\mathrm{C} 18-\mathrm{C} 19$

$\mathrm{C} 16-\mathrm{C} 18-\mathrm{C} 19-\mathrm{C} 20$

$\mathrm{C} 16-\mathrm{C} 18-\mathrm{C} 19-\mathrm{C} 21$

$\mathrm{C} 17-\mathrm{C} 12-\mathrm{C} 13-\mathrm{C} 14$

$\mathrm{C} 17-\mathrm{C} 12-\mathrm{C} 21-\mathrm{C} 19$

$\mathrm{C} 17-\mathrm{C} 12-\mathrm{C} 22-\mathrm{N} 2$

$\mathrm{C} 17-\mathrm{C} 16-\mathrm{C} 18-\mathrm{C} 19$

$\mathrm{C} 18-\mathrm{C} 16-\mathrm{C} 17-\mathrm{C} 12$

$\mathrm{C} 18-\mathrm{C} 19-\mathrm{C} 20-\mathrm{C} 14$

$\mathrm{C} 18-\mathrm{C} 19-\mathrm{C} 21-\mathrm{C} 12$

$\mathrm{C} 20-\mathrm{C} 14-\mathrm{C} 15-\mathrm{C} 16$

$\mathrm{C} 20-\mathrm{C} 19-\mathrm{C} 21-\mathrm{C} 12$

$\mathrm{C} 21-\mathrm{C} 12-\mathrm{C} 13-\mathrm{C} 14$

$\mathrm{C} 21-\mathrm{C} 12-\mathrm{C} 17-\mathrm{C} 16$

$\mathrm{C} 21-\mathrm{C} 12-\mathrm{C} 22-\mathrm{N} 2$

$\mathrm{C} 21-\mathrm{C} 19-\mathrm{C} 20-\mathrm{C} 14$

$\mathrm{C} 22-\mathrm{C} 12-\mathrm{C} 13-\mathrm{C} 14$

$\mathrm{C} 22-\mathrm{C} 12-\mathrm{C} 17-\mathrm{C} 16$

$\mathrm{C} 22-\mathrm{C} 12-\mathrm{C} 21-\mathrm{C} 19$
107.7

$-56.6(3)$

$-59.2(2)$

$60.3(2)$

$-59.1(2)$

$58.9(2)$

$-58.5(2)$

$60.1(2)$

$-59.6(2)$

$-60.3(2)$

$59.5(2)$

$60.3(2)$

$60.1(2)$

$-59.1(2)$

$59.5(2)$

$-60.4(2)$

$58.4(2)$

$-59.4(2)$

$64.6(2)$

$60.3(2)$

$-59.9(2)$

$-60.4(2)$

60.7 (2)

$-59.8(2)$

$-59.1(2)$

$-59.4(2)$

$58.9(2)$

$-177.13(16)$

59.0 (2)

$-176.66(16)$

$176.07(15)$

$179.66(15)$

Symmetry code: (i) $-x+1,-y+1,-z+1$.

Hydrogen-bond geometry $\left(\AA,{ }^{\circ}\right)$

\begin{tabular}{lllll}
\hline$D-\mathrm{H} \cdots A$ & $D-\mathrm{H}$ & $\mathrm{H} \cdots A$ & $D \cdots A$ & $D-\mathrm{H} \cdots A$ \\
\hline $\mathrm{O} 1-\mathrm{H} 1 D \cdots \mathrm{Cl} 2^{\mathrm{ii}}$ & $0.82(3)$ & $2.48(3)$ & $3.295(2)$ & $175(3)$ \\
$\mathrm{O} 1-\mathrm{H} 1 E \cdots \mathrm{Cl} 1$ & $0.91(4)$ & $2.36(4)$ & $3.265(2)$ & $180(3)$ \\
$\mathrm{N} 1-\mathrm{H} 1 A \cdots \mathrm{Cl} 2^{\mathrm{iii}}$ & $0.85(3)$ & $2.33(3)$ & $3.161(2)$ & $163(2)$ \\
$\mathrm{N} 1-\mathrm{H} 1 B \cdots \mathrm{O} 2$ & $0.89(3)$ & $2.10(3)$ & $2.867(3)$ & $144(2)$ \\
$\mathrm{N} 1-\mathrm{H} 1 C \cdots \mathrm{Cl} 1$ & $0.98(3)$ & $2.19(3)$ & $3.152(2)$ & $166(2)$ \\
$\mathrm{N} 2-\mathrm{H} 2 C \cdots \mathrm{Cl} 2^{\mathrm{ii}}$ & $0.86(2)$ & $2.31(3)$ & $3.166(2)$ & $172(2)$ \\
$\mathrm{N} 2-\mathrm{H} 2 D \cdots \mathrm{Cl} 1$ & $0.86(3)$ & $2.48(3)$ & $3.171(2)$ & $138(2)$ \\
$\mathrm{N} 2-\mathrm{H} 2 E \cdots \mathrm{Cl} 2$ & $0.90(3)$ & $2.30(3)$ & $3.181(2)$ & $166(2)$
\end{tabular}

Symmetry codes: (ii) $-x+2,-y,-z+1$; (iii) $x-1, y, z$. 